\title{
ANEMIA EN LA POBLACIÓN PEDIÁTRICA DEL PERÚ
}

ANEMIA IN THE PEDIATRIC POPULATION OF PERU

\author{
MESA REDONDA, INSTITUTO NACIONAL DE SALUD DEL NIÑO: \\ Carlos del Águila-Villar, Eliana Chávez-Tejada, Alberto Romero-Guzmán, Zulema Román-Blas, Oswaldo Núñez-Almache
}

\section{INTRODUCCIÓN}

En el mundo la deficiencia de hierro afecta a 2000 millones de personas y de ellos 1,600 millones tienen anemia por deficiencia de hierro ${ }^{1,2}$.

De ellos un $47,4 \%$ son pre-escolares, $25,4 \%$ son escolares, $41,8 \%$ son gestantes y $30,2 \%$ son mujeres en edad fértil2.

La severidad del problema es establecida por la OMS según el grado de prevalencia, y se considera severo si afecta a más del $40 \%$ de la población, moderado si afecta entre el 20 y $40 \%$ de la población, y leve si afecta a menos del $20 \%{ }^{2}$.

En América Latina se reporta que afecta a 50\% de niños menores de 5 años, $75 \%$ de niños menores de 2 años; 10 a $30 \%$ de mujeres en edad reproductiva y al 40 a $70 \%$ de las mujeres embarazadas ${ }^{3}$.

En el Perú existe sub registro de la anemia. MINSA reporta el diagnóstico de anemia ferropénica en sólo $2.1 \%$ de las consultas ambulatorias ${ }^{4}$, mientras la encuesta ENDES 2015 reporta que el problema es serio para población de niños más pequeños. Según esta última publicación de INEl, el 31\% de los niños menores de 5 años encuestados tiene anemia, y está presente en casi $60 \%$ de menores de 2 años, en $20,7 \%$ de las mujeres entre 15 y 49 años, y en $28 \%$ de las gestantes 5 .

Hay una asociación significativamente alta entre anemia materna y desarrollo del niño en el primer año de vida ; la deficiencia de hierro en los primeros 3 años de vida, afecta la formación de la mielina ${ }^{7}$, y en lactantes anémicos el tiempo de conducción del estímulo nervioso es más largo ${ }^{8}$. Más aún, los lactantes anémicos continúan mostrando un tiempo de conducción más largo después de recibir un tratamiento prolongado con hierro oral, ocasionando trastornos de aprendizaje, de productividad y emocionales ${ }^{9}$, alteraciones que persisten hasta la etapa adulta, inclusive después de suplementar y corregir el déficit de hierro ${ }^{10}$. Lo grave es que hay evidencia que el déficit psicomotor no es corregible si la anemia ferropénica ocurre en los 2 primeros años de vida ${ }^{11}$.

Un estudio realizado en Perú en 2012 calculó que la anemia le cuesta al Gobierno peruano 2,777 millones de soles equivalente al $\mathbf{0 , 6 2 \%}$ del $\mathrm{PBI}$, incluyendo costos para le economía (Costos por pérdida cognitiva, por pérdida de escolaridad y por pérdida de la productividad) y costos para el estado (costos por atención de parto prematuro, por años de repitencia, por tratamiento de los niños y de las gestantes) ${ }^{12}$.

En consecuencia podemos afirmar que afrontamos un serio problema de salud pública, y que persiste pese a los continuos esfuerzos realizados por el estado para controlarlo.

Moderador Dr. Carlos del Águila: Anemia ferropénica en el niño como problema de salud pública en el Perú.

\section{DISCUSIÓN GENERAL}

El Instituto Nacional de Salud del Niño es la primera institución estatal dedicada a la salud de los niños del país, y en concordancia con esta misión, la Oficina Ejecutiva de Apoyo a la Investigación y Docencia Especializada, organizó y coordinó una Mesa Redonda dedicada a tratar un tema que afecta a nuestro país: "Anemia ferropénica en el niño como problema de salud pública en el Perú". Esta actividad se llevó a cabo el miércoles 25 de mayo pasado en el Aula San Martín del INSN.

La Mesa Redonda fue moderada por el Dr. Carlos del Águila Villar Pediatra Endocrinólogo y Jefe del Servicio de Endocrinología Pediátrica; participaron como panelistas médicos del INSN: el Dr. Alberto Romero Guzmán, Médico Pediatra Jefe del Servicio de Medicina B, la Dra. Eliana Chávez Tejada, Responsable del Programa CRED, la Dra. Zulema Román Blas Hemató- 
loga Pediatra del Servicio de Hematología y el Dr. Oswaldo Núñez Almache Pediatra Endocrinólogo del Servicio de Endocrinología Pediátrica.

A ella fueron convocados todos los médicos asistentes, residentes e internos del INSN.

La reunión se inició con la participación del Dr. Del Águila quien realizó una breve introducción al tema "Anemia ferropénica en el niño como problema de salud pública en el Perú"; el ser humano en su evolución se ha ido adaptando al medio y específicamente en el Perú el proceso de migración y adaptación a medio ambientes diferentes, ha ido influenciando y modificando sus hábitos nutricionales, con resultados negativos como obesidad y deficiencias nutricionales incluida la deficiencia de hierro. Ello es palpable aún en el siglo XXI en el difícil territorio peruano, en cuyos habitantes prevalecen aún ciertas patologías, siendo la anemia una de ellas, la cual además de ser un problema ligado a la malnutrición, condiciona un problema serio de Salud Pública, con elevado costo social y económico. Los intentos del gobierno para corregir esta condición son múltiples con resultados parciales. El pediatra debe asumir su rol como especialista y participar activamente en los lineamientos para solucionarlo.

El Dr. Alberto Romero presentó el tema "Anemia en la población pediátrica del Perú", y recalcó la elevada prevalencia que tiene la anemia en la población pediátrica más vulnerable y si bien es problema de todo el país, hay regiones en el que es más severo.

Informó sobre los valores de corte de hemoglobina que debe servir para el diagnóstico, haciendo enfasis en que estos valores deberían adecuarse de acuerdo a la altitud sobre el nivel del mar en la que habita el niño, y que ésta influye en el status socio económico cultural. Pero tan importante como valorar la anemia es evaluar la deficiencia de hierro, precursora de la anemia, que en los niños conlleva a alteración irreversible del sistema nervioso central, con impacto en el desarrollo neurosensorial, motor, cognitivo y emocional. Mencionó la importancia que tiene la intervención del pediatra, sobre todo para la detección temprana de la deficiencia de hierro y la anemia y que el médico debiera utilizar para una evaluación mucho más apropiada los parámetros ferritina, hierro sérico, saturación de transferrina y hemoglobina, con la finalidad de detectar e intervenir apropiadamente en estadios tempranos de deficiencia de hierro que podrían llevar al desarrollo de la anemia y sus nefastas consecuencias.

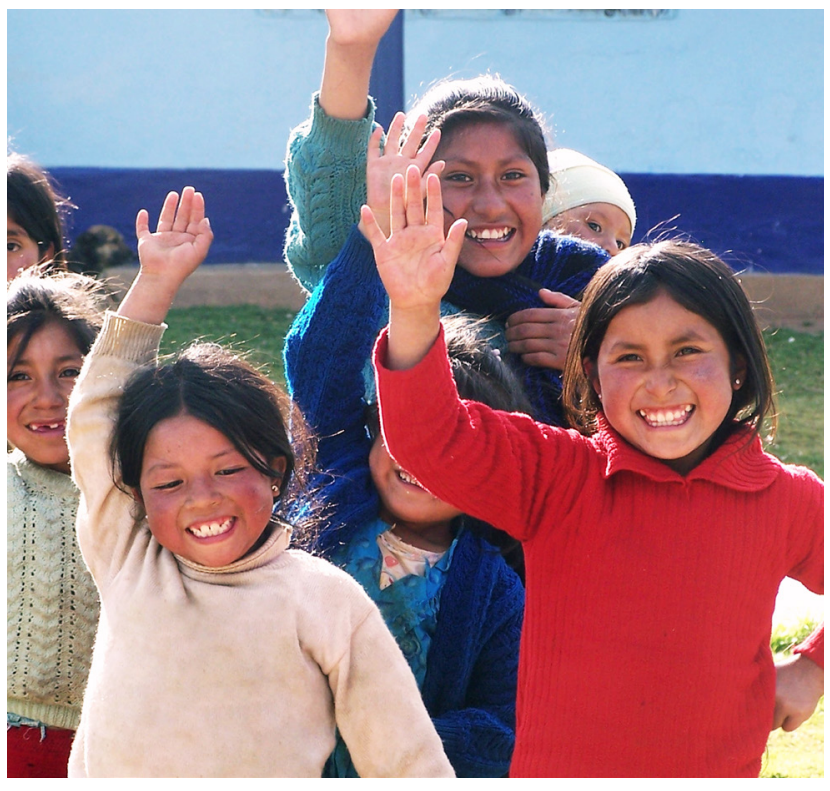

Por su parte la Dra. Chávez, resumió los fundamentos y actividades que el CRED desarrolla en el INSN y cuáles son las objeciones a las medidas que en el Programa se llevan a cabo, resaltando que el objetivo es disminuir la tasa de morbilidad y mortalidad por causas evitables mediante la prevención e identificación oportuna de los problemas que afectan a los niños menores de 5 años. SI bien es un programa para aplicar en establecimientos de salud Nivel I, su aplicación en el INSN como un Programa CRED complejo, se explica por la asistencia al INSN de niños portadores de patologías complejas que no son atendidas en los centros básicos. El Programa CRED tiene como uno de sus objetivos evaluar el estado de anemia de la población de menores de 5 años, para lo cual el instrumento es la medición de la hemoglobina, y en base a la cual se aplican medidas preventivas o de tratamiento con micronutriente y hierro oral. La Dra. Chávez explica la alta prevalencia de anemia en la población objetivo debido a

- Causas del Sistema (bajo presupuesto, Inaccesibilidad del sistema de salud, Programa CRED recae preponderantemente en personal de enfermería, Ausencia de estrategias efectivas para prevención y tratamiento, Propuestas de suplementación de hierro que no resuelven el problema (Chispitas), Hierro proveído por SIS es sulfato ferroso, Ausencia de fortificación de otros alimentos, Circulo de pobreza/desnutrición/ enfermedad no resuelto.

- Causas asociadas al quehacer médico (Atención especializada Pediátrica insuficiente, Desconocimiento de real magnitud epidemiológica del problema, Conocimiento inadecuado/insufi- 
ciente de importancia del hierro, Atención Médica curativa y no preventiva, Pesquisa inusual o insuficiente para anemia, Prejuicios clínicos no advertidos, Mala interpretación de exámenes solicitados, No criterios claros de Referencia al especialista, Prescripción inadecuada, Ausencia de control al termino del tratamiento, No se imparte educación nutricional, médicos Generales y pediatras rechazan la práctica CRED.

- Causas inherentes a la madre (Madre con bajo nivel económico y bajo nivel educativo, Madre adolescente, primigesta, Anemia gestacional no tratada o de tratamiento insuficiente, Ingesta insuficiente de alimentos con hierro en el embarazo, Adherencia inadecuada al tratamiento en el embarazo, Lactancia materna no exclusiva e insuficiente, Madre que trabaja y subroga el cuidado del bebe a terceros, Alimentación complementaria tardía y no adecuada, Madre que no busca asesoría del sistema de salud, Resistencia de los padres a la toma de muestra, Madre con actitudes y creencias inadecuadas, Mala adherencia al tratamiento).

- Creencias erróneas ("El bebe no tiene dientes" , "Recién al año puede comer carne" , "Las Carnes rojas hacen daño" , "Las carnes son difíciles de masticar y digerir ", "somos poco de comer carnes ", "la espinaca puede prevenir y curar la anemia", "Extracto de Betarraga es útil contra la anemia", "Las lentejas pueden resolver la anemia", "Sangre de grado".

- Dificultades debidas al tipo de hierro ("Será suficiente un mes", "El hierro Pigmenta los dientes ", "El hierro le causa caries", "Es difícil de administrar ", "El sabor es desagradable", " Produce Estreñimiento", " Produce diarrea", " El hierro facilita las infecciones ", "Su estomaguito se carga y las heces se negrean "," Alto costo "

La Dra. Chávez concluye afirmando que la educación a la familia sería una intervención primordial para resolver estos problemas y remarcó la importancia de la intervención del pediatra en la evaluación de los niños y como educador de la familia.

La Dra. Zulema Román informó que en el Servicio de Hematología, en 5 años (2011 a 2015) la mitad de las consultas fueron por anemia y que un tercio de ellas fueron anemias ferropénicas (un total de 3170 niños), generando sobrecarga en la atención en consultorios; con una adecuada evaluación con historia, examen clínico y parámetros de laborato- rio, estos casos podrían ser evaluados por pediatras generales o las especialidades pediátricas a las cuales acuden los pacientes del INSN.

El Dr. Oswaldo Núñez comentó sobre las diferentes estrategias diseñadas por el estado para solucionar este grave problema, y que si bien la estrategia de aporte de micronutrientes (MMN "Chispita") es un buen modelo preventivo en niños con niveles de hemoglobina normal y adecuado depósito de hierro, resulta insuficiente cuando se utiliza para tratar la deficiencia de hierro y la anemia ferropénica. De otro lado el sulfato ferroso puede ocasionar eventos adversos que obliguen a suspender el tratamiento o provocar falta de adherencia al tratamiento conllevando a ineficacia de la intervención; en estos casos, la inclusión del hierro polimaltosado, según experiencia obtenida con investigación nacional, abre las puertas a un mejor adherencia y por tanto, a resultados más favorables. En ese sentido también recalcó la labor fundamental que debe asumir el pediatra general como director del equipo médico que debe conducir las diversas estrategias que ayuden a resolver el problema, y que, si adicionalmente el estado ha aprobado incluir otras sales de hierro, queda en el médico, según la evidencia científica, decidir cuál es la mejor aceptada y ayudaría a solucionar de manera eficaz la anemia ferropénica.

Esta Mesa Redonda ha sido de gran utilidad para revisar un severo problema de la salud de los niños del Perú y para que los profesionales de salud del INSN incrementen su conciencia sobre el tema. Asimismo sirva como punto de partida para una revisión más profunda de la anemia a nivel de cada departamento y servicio de la institución, y la generación de guías para su apropiado diagnóstico y manejo, en el ámbito local y nacional, lo cual redundará en el progreso socio económico del país.

Breña, Mayo 2016.

\section{Moderador}

- Dr. Carlos del Águila Villar CMP 18157 - RNE 7987. Pediatra, Jefe del Servicio de Endocrinología en el INSN.

\section{Participantes}

- Dra. Eliana Chávez Tejada CMP 27215 - RNE 14796. Pediatra, Responsable del Programa CRED en el INSN. Programa CRED y anemia infantil.

- Dr. Alberto Romero Guzmán CMP 27435 - RNE 14389. Pediatra, Médico Jefe del Servicio de Me- 
dicina B del INSN. Anemia infantil: el punto de vista del pediatra.

- Dra. Zulema Román Blas CMP 30161 - RNE 13018. Hematóloga del Servicio de Hematología del INSN. Anemia infantil: ¿Cuándo debo derivar al hematólogo al paciente anémico?

- Dr. Oswaldo Núñez Almache CMP 22698 - RNE 11845 - RNE 25392. Pediatra, Endocrinólogo Pediatra. Médico del Servicio de Endocrinología del INSN. Anemia infantil: experiencia sobre eficacia y seguridad de la terapeutica en pediatria.

\section{Agradecimientos Especiales:}

Al Dr. Guillermo Arribasplata.
Financiamiento: Autofinanciado.

Conflicto de interés: Los autores declaran no tener conflictos de interés en la publicación de este artículo.

Recibido: 31 de Mayo de 2016

Aprobado: 01 de Julio de 2016

\section{REFERENCIAS BIBLIOGRÁFICAS}

1. Miller, J. Cold Spring Harb Perspect Med. 2013 Jul; 3(7): a011866.

2. WHO Global Database of Anaemia Prevalence 1993 -2005. Publicado 2008

3. McLEan et al. 2008. Public Health Nutrition: 12(4), 444-454).

4. http://www.app.minsa.gob.pe/bsc/Detalle_IndBSC.asp?lcind=5\&l-

cobj=1\&lcper $=1 \& \mid c f r e g=4 / 3 / 2016$

5. Perú: Encuesta Demográfica y de Salud Familiar INEI 2015

6. Colomer J et al. Paediatr Perinat Epidemiol 1990;4:196-204
7. Mittal et al. Indian Journal of Clinical Biochemistry, 2003, 18 (1) 111-116

8. Roncagliolo et al. Am J Clin Nutr 1998 68: 3 683-90

9. Lozoff, B. et al. J Pediatr. 2008 May ; 152(5): 696-702.33

10. Beard et al. J Nutr. 2007 Feb;137(2):524S-530S

11. Food and Nutrition Bulletin, vol. 28, no. 4 (supplement) (c) 2007, The United Nations University.

12.Alcázar L., Impacto económico de la anemia en el Perú /Lima: GRADE; Acción contra el Hambre, 2012),

Consulte la Versión Electrónica de la Revista:

Facultad de Medicina Humana Universidad Ricardo Palma

http://revistas.urp.edu.pe/ojs/index.php/RFMH
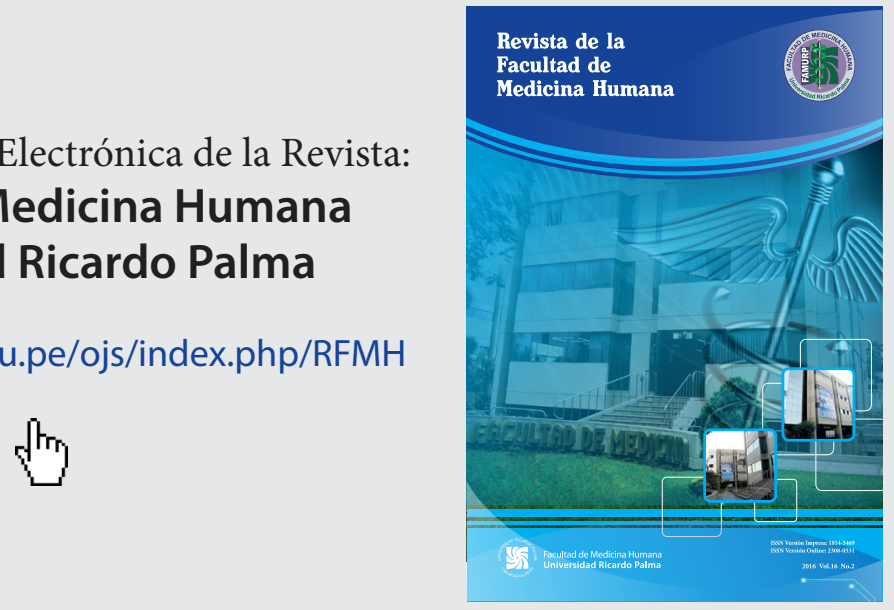